\title{
Phase Synchronization of Two Anharmonic Nanomechanical Oscillators
}

\author{
Matthew H. Matheny, Matt Grau, Luis G. Villanueva, Rassul B. Karabalin, M. C. Cross, and Michael L. Roukes* \\ Kavli Nanoscience Institute and Departments of Physics, Applied Physics, and Bioengineering, \\ California Institute of Technology, Pasadena, California 91125, USA
}

(Received 6 May 2013; published 6 January 2014)

\begin{abstract}
We investigate the synchronization of oscillators based on anharmonic nanoelectromechanical resonators. Our experimental implementation allows unprecedented observation and control of parameters governing the dynamics of synchronization. We find close quantitative agreement between experimental data and theory describing reactively coupled Duffing resonators with fully saturated feedback gain. In the synchronized state we demonstrate a significant reduction in the phase noise of the oscillators, which is key for sensor and clock applications. Our work establishes that oscillator networks constructed from nanomechanical resonators form an ideal laboratory to study synchronization - given their high-quality factors, small footprint, and ease of cointegration with modern electronic signal processing technologies.
\end{abstract}

PACS numbers: 05.45.Xt, 05.40.Ca, 07.05.Dz, 85.85.+j

Synchronization is a ubiquitous phenomenon both in the physical and biological sciences. It has been observed to occur over a wide range of scales-from the ecological [1], with oscillation periods of years, to the microscale [2], with oscillation periods of milliseconds. Although synchronization has been extensively studied theoretically [3-5], relatively few experimental systems have been realized that provide detailed insight into the underlying dynamics. Here we show that oscillators based on nanoelectromechanical systems (NEMS) can readily enable the resolution of such details, while providing many unique advantages for experimental studies of nonlinear dynamics [6-8]. In addition, nanomechanical systems might prove useful for exploring quantum synchronization $[9,10]$.

Nanomechanical oscillators also have been exploited for a variety of applications [11-13]. In particular, nanoscale mechanics exhibits enhanced nonlinearity $[14,15]$ and tunability $[16,17]$, which has been used to suppress feedback noise $[18,19]$ and create new types of electromechanical oscillators [20-22]. These oscillators may find application as mass [23], gas [24,25], or force sensors [26], without the need of an external frequency source.

Building frequency sources from arrays of NEMS may yield enhanced applicability, but is challenging. For example, statistical deviations in batch fabrication inevitably lead to undesirable array dispersion [24]. If an array has appreciable frequency dispersion, global sensor responsivity gets reduced. However, if the elements of the array are made into a self-sustained oscillators and synchronized with one another, then the array responsivity will recover due to a reduction in phase noise [3]. Since NEMS have numerous applications, and are useful in studying nonlinear dynamics, we set an important milestone by demonstrating synchronization in nanomechanical systems.

There are previous reports of synchronization in microor nanomechanical systems. However, these do not, in fact, demonstrate the phenomenon as conventionally defined [3] - that is, the phase locking of weakly coupled selfsustained oscillators. Shim et al. [27] reported synchronization of the driven excitations in coupled resonators, not self-sustained oscillators. Zhang et al. [28] reported self-sustained oscillations excited by radiation pressure in optomechanical resonators, coupled through the evanescent optical field. However, the model and data presented in Zhang et al. reflect strong coupling [29], with the energy to excite the oscillations equal to the energy to couple the devices. This strong coupling inevitably leads to confusion between synchronization of individual oscillators and the excitation of a single coupled mode.

Our experiment is designed to unambiguously demonstrate canonically defined synchronization with a pair of weakly coupled oscillators. This is accomplished by employing an additional feedback loop, separate from the feedback loop necessary to sustain oscillations, to couple the resonant devices. This coupling can be modified via analog electronics, allowing full control of all relevant parameters. Importantly, it can set to a value where the coupling is a weak perturbation on the individual oscillator dynamics. Since all of these parameters are carefully calibrated, we can make quantitative comparisons with theory, yielding an ideal platform to elucidate synchronization. Our implementation is scalable to thousands of devices through standard methods of large-scale integration. To show the applicability of synchronized NEMS, we measure the phase noise of the oscillators, and demonstrate the reduction in phase noise theoretically expected from noise averaging.

We describe our system with a set of equations similar to the model theoretically examined by Aronson et al. [30], except that here our oscillation amplitude is not constrained by nonlinear dissipation, but rather by amplifier saturation. We scale the amplitude by the level of saturation and examine the system dynamics in slow time, $T \sim Q * t * \omega_{0}$, 
where $Q$ is the quality factor of the driven response of the resonators and $\omega_{0}$ the linear resonance frequency of the NEMS device when under driven excitation, and $t$ is the real time in seconds. In the slow time dynamics, feedback loop time delays are represented by a phase shift. The resulting equations for the amplitudes $a_{1,2}$ for each oscillator and phase difference $\varphi$ between them are [29]

$$
\begin{gathered}
a_{1,2}^{\prime} \equiv \frac{d a_{1,2}}{d T}=-\frac{a_{1,2}}{2}+\frac{1}{2} \mp \frac{\beta}{2} a_{2,1} \sin \varphi \\
\varphi^{\prime} \equiv \frac{d \varphi}{d T}=\Delta \omega-\left(a_{1}^{2}-a_{2}^{2}\right)\left(\alpha-\frac{\beta}{2 a_{1} a_{2}} \cos \varphi\right),
\end{gathered}
$$

where,-+ corresponds to $a_{1}, a_{2}$, respectively. Here $\Delta \omega$ is the difference between the resonant frequencies of the devices, $\alpha$ is the measure of frequency pulling (which is the increase in frequency proportional to the square of the amplitude), and $\beta$ is the coupling strength. Note that our coupling here is not dissipative, but reactive, in contrast to most studies of synchronization to date [31]. Reactive coupling inevitably leads to the amplitudes playing a key role in the synchronization, as previously shown theoretically [31,32]. The parameters $\Delta \omega, \alpha$, and $\beta$, which we call the synchronization parameters, set the dynamics of the system: the stable fixed points of Eqs. (1) and (2), for example, yield synchronized states. These parameters are expressed in units of the devices' resonance line width, $\omega_{0} / Q$.
To construct an experiment with independent control of the synchronization parameters we use the setup shown in Fig. 1. The NEMS devices are two piezoelectrically actuated, piezoresistively detected [14], doubly clamped beams $10 \mu \mathrm{m}$ long, $210 \mathrm{~nm}$ thick, and $400 \mathrm{~nm}$ wide. In the oscillator loop, the signal is strongly amplified (gain stage, $g$ ) into a diode limiter (saturation stage, $s$ ) in order to ensure the feedback signal to the beam is of constant magnitude. Therefore, the feedback signal is a strongly nonlinear function of the device displacement [19]. On the other hand, the coupling loop is kept linear; the feedback is directly proportional to the displacement over the full range of experimental values. For the oscillator loop, the signal is fed back in phase with the beams velocity. For the coupling loop, this signal is fed into the beams in phase with the displacement. This causes the coupling loop to be reactive and the oscillator loop to be dissipative. The synchronization parameters are each controlled by a dc voltage. Adjusting oscillator feedback saturation controls frequency pulling $\alpha$, adjusting coupling feedback amplitudes controls coupling $\beta$, and adjusting piezovoltage controls frequency detuning $\Delta \omega$ [29].

This system is designed to be integrable within CMOS technologies. The system consists of transistor amplifiers, saturation diodes, direction couplers (capacitors), and phase shifters. Here we use adjustable attenuators; these may also be implemented with adjustable amplifiers. The phase shifters can be implemented with fixed resistancecapacitance filters. However, we note that if we measure the piezoelectric response in addition to the piezoresistive response, we are able to directly capture both the in-phase and out-of-phase response of the oscillators. Since all parameters are controlled with dc voltages,

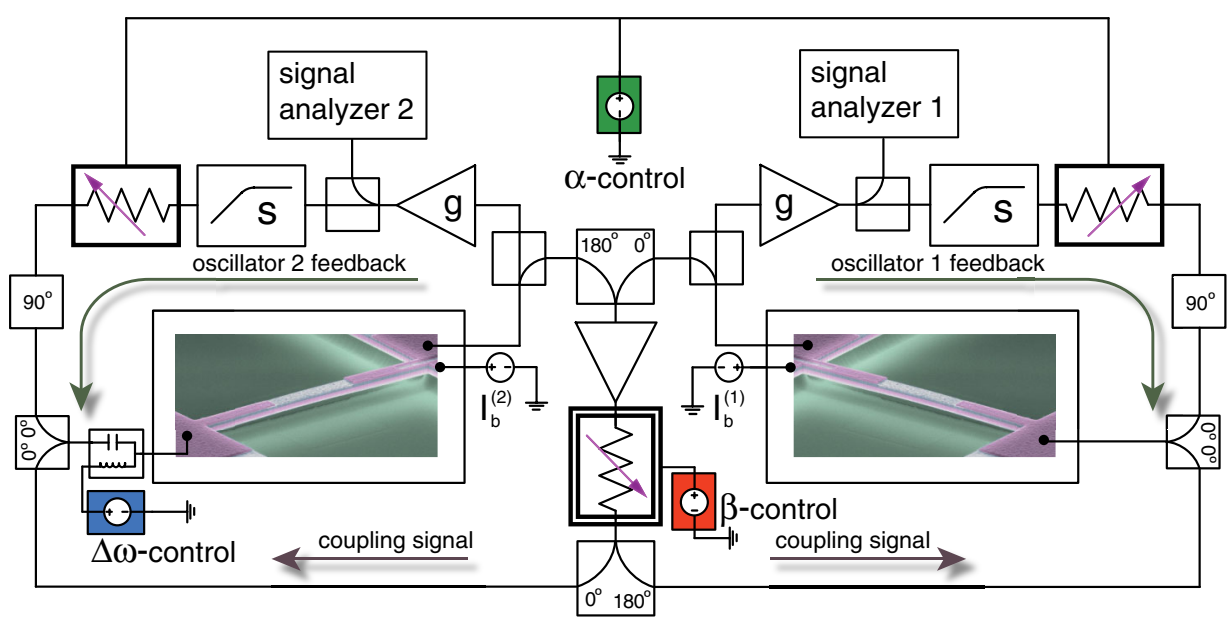

FIG. 1 (color online). Simplified circuit schematic for experiment. Each NEMS resonator (colored SEM micrograph) is embedded in two feedback loops: one is used for creating oscillations in each resonator, and the other creates coupling between the oscillators. The attenuators after each limiter (single heavy line boxes) sets the level of oscillation, and constitutes a means to control the frequency pulling. In the coupling loop the signal is amplified so that an attenuator (double heavy line boxes) adjusts the signal level in the common loop, thereby setting the coupling strength. The frequency difference is controlled by adjusting the stress induced in the left resonator by the piezovoltage. 
our system offers the possibility of massive arrays with individual control over constituent elements.

We begin by looking at the small coupling limit, with $\beta<0.1$, where experiment can be compared to analytical predictions. In that case, the amplitudes of the two oscillators stay near unity, so Eqs. (1) and (2) become

$$
\begin{gathered}
a_{1,2}=1 \mp \beta \sin \varphi, \\
\varphi^{\prime}=\Delta \omega+4 \alpha \beta \sin \varphi,
\end{gathered}
$$

where Eq. (4) is the Adler equation [33]. Note that even though Eq. (4) is of the same form as the one Adler used to study injection locking, it describes the mutual synchronization of two oscillators [29]. When the oscillators are unsynchronized, the solution to Eq. (4) can be expressed as

$$
\varphi^{\prime}=\sqrt{\Delta \omega^{2}-(4 \alpha \beta)^{2}} .
$$

In the synchronization regime $\left(\varphi^{\prime}=0\right)$, as the amplitudes stay near unity, a linear relationship between the oscillation amplitudes and the frequency difference is found from Eqs. (3) and (4),

$$
\frac{\partial a_{1,2}}{\partial \Delta \omega}= \pm \frac{1}{4 \alpha},
$$

where 1,2 corresponds to,+- , respectively. Equation (6) holds explicitly in the synchronization regime.

In Fig. 2, $\varphi^{\prime}$ is the oscillator frequency difference in units of the resonance width. The plots show synchronization between the two coupled oscillators, with remarkable agreement between Eqs. $(5,6)$ and the experiment. The oscillator amplitudes change in order to adjust the oscillator frequencies, demonstrating the importance of frequency pulling in reactively coupled oscillators.

In addition to control of the detuning through a wide range of values (shown in Fig. 2), we are able to modify both the frequency pulling and coupling, to study the parameter space for synchronization. Figure 3 shows the synchronization parameter space for three levels of fixed detuning $(\Delta \omega=0.6,1,2)$ as coupling and frequency pulling $\alpha$ are varied. The red border is the data with attractive (ATT) coupling $[\beta<0$ in Eqs. $(1,2)]$ and green with repulsive (REP) coupling $[\beta>0$ in Eqs. $(1,2)]$. These lines represent the boundaries of the transition between synchronized and unsynchronized states when sweeping to higher values of coupling, i.e., from left to right in Fig. 3. This transition is defined by a change to a measured oscillator frequency difference $\varphi^{\prime}<0.05$.

In general, analytical solutions to Eqs. (1) and (2) cannot be found. Therefore, we perform two numerical studies and compare them to the experiment. We perform a linear stability analysis (LSA) [34] of Eqs. (1) and (2) with the orange and purple dashed lines in Fig. 3 showing the stability boundaries. The LSA boundaries define only where the synchronized states are stable, and so there may be unsynchronized stable states coexisting within these boundaries.

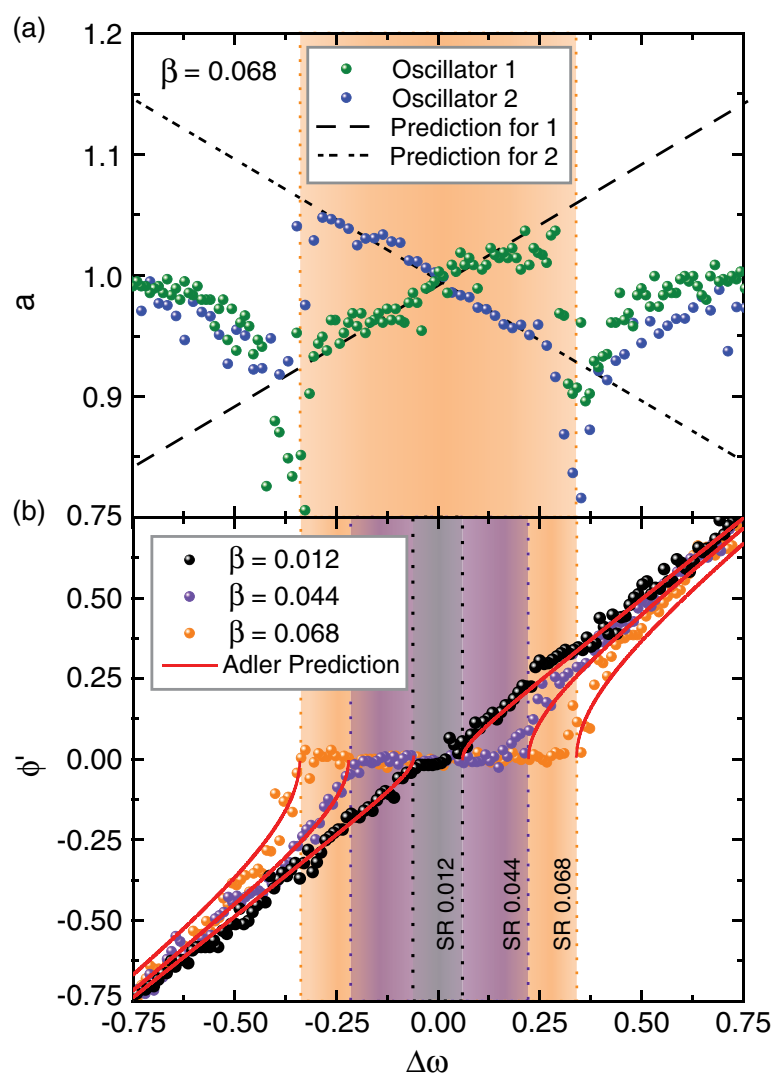

FIG. 2 (color online). Synchronization in the limit of small coupling described by Eqs. (5) and (6) with a frequency pulling $\alpha=1.25$. (a) Experimental data (points) are compared against theoretical predictions (lines) for the amplitudes of the two oscillators as the system moves through synchronization; the dependence upon detuning $\Delta \omega$ for a coupling of $\beta=0.068$ is shown. The synchronization regime is shown by orange shading. (b) Data and predictions for the frequency difference $\varphi^{\prime}$ for three different values of coupling. The set of data with the largest value of coupling $\beta=0.068$ corresponds to the amplitude data from the upper plot. Frequency locking (synchronization regime) is shown where values $\varphi^{\prime}=0$ occur. SR 0.012, SR 0.044, SR 0.068 denote the synchronization regimes (shaded regions) for the three couplings.

We also perform a time domain simulation of Eqs. (1) and (2), (with $\beta>0$ ) using initial conditions of amplitudes fixed at 1 and random phases. At each point in paramater space this time domain simulation gives a basin of attraction for stabilizing in either an unsynchronized or a synchronized state (from an initially unsynchronized state). For each value of the parameters plotted in Fig. 3, we run 100 such simulations and assign a synchronization value of 0 for unsynchronized and 1 for synchronized. The average value of these 100 simulations is represented by a linear gradient between white and blue for 0 and 1, respectively. Note that the parameters space which synchronizes (blue) lies within the LSA-1 boundaries, and there is a slight increase in probability for synchronizing inside the border of the LSA-2 boundary. However, the initial amplitudes of our time-domain simulation are not random. The 


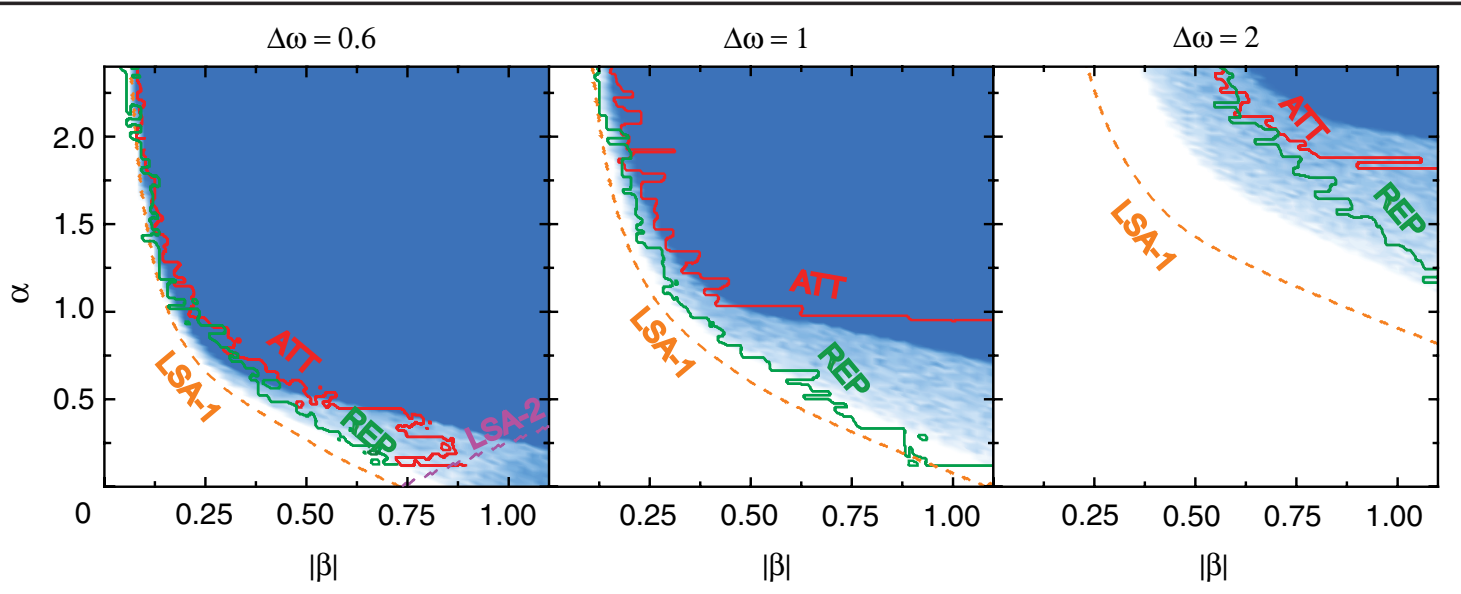

FIG. 3 (color online). Experimentally measured synchronization space as a $\beta$ and $\alpha$ for $\Delta \omega=0.6,1,2$. The basins of attraction, found from the time domain simulation of Eqs. (1) and (2), are shown by the gradient between white and blue, and correspond to the average number of times the simulation synchronized under 100 random initial phases. All lines show boundaries with respective regions to the right of the line. The green solid line (REP) is the experimental boundary for the transition from unsynchronized to the synchronized state for repulsive coupling. The red solid line (ATT) is the experimental boundary for the transition from the unsynchronized to the synchronized state for attractive coupling. The experimental synchronized state is defined as $\varphi^{\prime}<0.05$ (in units of the resonator width). The orange dashed line (LSA-1) depicts the predicted (linear stability analysis) boundary for which at least one synchronized state is stable. Similarly, the purple dashed line (LSA-2) bounds the space for which both synchronized states are stable.

amplitudes are fixed to unity, in accord with the experiment. This causes the area bordered by the LSA to be larger than the synchronized regions shown by the time domain simulation.

We can distinguish two different "borders" in the basins of attraction. The first is moving from completely white to lightly shaded blue. The second is moving from lightly shaded blue to completely dark blue. Synchronization between these borders is determined by the initial phase of the simulation. The experimental boundaries (red and green lines) seem to correspond to these borders from the time-domain simulation. However, Eqs. (1) and (2) are completely symmetric upon exchange of $\beta \mapsto-\beta$, since synchronization will occur for $\varphi \mapsto \varphi+\pi$. Thus, given an initial random phase difference between the oscillators, these two boundaries for positive and negative $\beta$ should overlap. On the other hand, the two time-domain simulation borders do correspond quite well to the experimental data. This could be accounted for if the initial phase difference of the oscillators is not completely random, but biased towards a particular phase. This may be due to higher order dynamics or experimental asymmetries.

We observe that at large detunings, asymmetries in saturation level or discrepancies in quality factor between the two oscillators tend to create larger disagreement between theory and experiment. This is due to the large coupling necessary in order to synchronize the oscillators, which magnifies the nonlinear behavior (and thus asymmetry) of the system. However, the close agreement of Figs. 2 and 3 show the generality and accuracy of our approach.

Finally, we explore the effect of synchronization on the phase noise. In Fig. 4, the green and blue spheres are the phase noise at $1 \mathrm{kHz}$ offset from the carrier frequency (a key figure of merit for the frequency source community [35]) plotted as a function of coupling for oscillators 1 and 2, respectively [29]. The red diamonds show the oscillator frequency difference $\varphi^{\prime}$ for comparison. As coupling is increased the phase noise at this offset initially increases (due to phase slipping between the oscillators) and then suddenly drops to $3 \mathrm{~dB}$ below the uncoupled noise level. The plot of the oscillator frequency difference indicates that the phase noise reduction occurs at the onset of synchronization. This corresponds to a phase noise reduction by factor of two, as predicted by theoretical estimates [3].

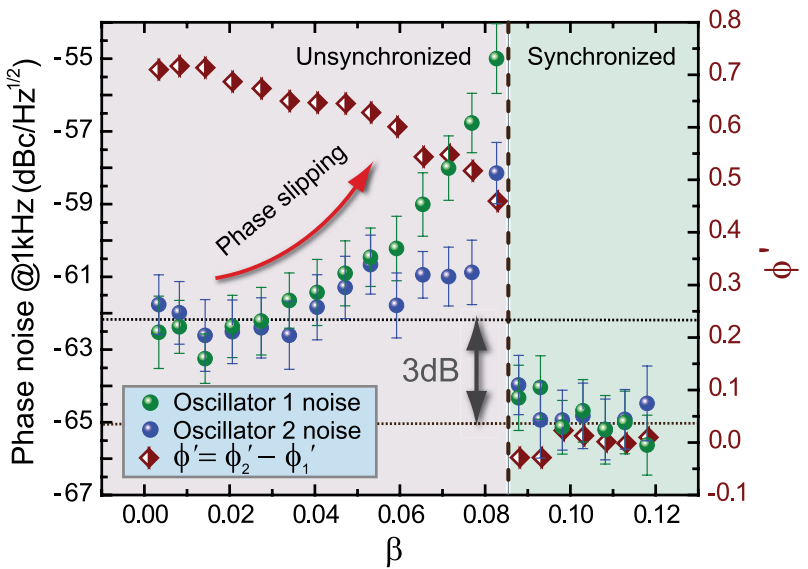

FIG. 4 (color online). Oscillator phase noise at $1 \mathrm{kHz}$ offset from carrier frequency (blue and green spheres, left axis) and oscillator frequency difference (red diamonds, right axis) as coupling is increased. At the value of coupling $\beta=0.086$ the oscillator frequency difference goes to zero and the phase noise for both oscillators decreases by $3 \mathrm{~dB}$, i.e., corresponding to reducing the phase noise by half. 
Our demonstration of the synchronization of two reactively coupled anharmonic NEMS oscillators shows excellent agreement with analytical and numerical modeling. We track not only the frequency difference, but also the individual amplitudes, important for a full multivariable description of the synchronization. These results highlight the importance of the oscillator amplitudes in synchronization for reactive coupling. Our work shows the potential of this system to examine nonlinear dynamics at the intermediate scale of discretization: full control of individual elements and tracking of large arrays. All of the components in these experiments can be realized using CMOS technology, implying that very large scale networks can be built using the precise technology of present-day semiconductor nanoelectronics and electronically tested with cointegrated state-of-the-art signal processing capabilities. The flexibility of this system permits creation of dissipative or reactive coupling in arbitrarily complex or completely random networks. Our experimental demonstration of reduced phase noise in the synchronized state marks an advance for detection of very weak phenomena using synchronized nanoscale sensor arrays.

We thank E. Kenig and X.L. Feng for discussions and P. Ivaldi, E. Defaÿ, and S. Hentz for providing us with the AlN/SOI material. M. C. Cross acknowledges financial support from the National Science Foundation Grants No. DMR-0314069 and No. DMR-1003337. L. G. V. acknowledges financial support from the European Commission (PIOF-GA-2008-220682).

In the process of going through review, synchronization was explored in a similar system [36].

*Corresponding author.

roukes@ caltech.edu

[1] B. Blasius, A. Huppert, and L. Stone, Nature (London) 399, 354 (1999).

[2] G. B. Ermentrout and D. Kleinfeld, Neuron 29, 33 (2001).

[3] A. Pikovsky, M. Rosenblum, and J. Kurths, Synchronization: A Universal Concept in Nonlinear Sciences (Cambridge University Press, 2003), Vol. 12.

[4] A. Tyrrell, G. Auer, and C. Bettstetter, in Proceedings of the 1st International Conference on Bio Inspired Models of Network, Information and Computing Systems (ACM, New York, 2006), p. 4.

[5] R. E. Mirollo and S. H. Strogatz, SIAM J. Appl. Math. 50, 1645 (1990).

[6] R. Lifshitz and M.C. Cross, Reviews of Nonlinear Dynamics and Complexity Vol. 1, (Wiley-VCH Verlag GmbH \& Co. KGaA, Weinheim, 2008).

[7] G. Heinrich, M. Ludwig, J. Qian, B. Kubala, and F. Marquardt, Phys. Rev. Lett. 107, 043603 (2011).

[8] C. A. Holmes, C. P. Meaney, and G. J. Milburn, Phys. Rev. E 85, 066203 (2012).

[9] A. Mari, A. Farace, N. Didier, V. Giovannetti, and R. Fazio, Phys. Rev. Lett. 111, 103605 (2013).
[10] N. Lörch, J. Qian, A. Clerk, F. Marquardt, and K. Hammerer.

[11] I. Mahboob, E. Flurin, K. Nishiguchi, A. Fujiwara, and H. Yamaguchi, Nat. Commun. 2, 198 (2011).

[12] O. Y. Loh and H. D. Espinosa, Nat. Nanotechnol. 7, 283 (2012).

[13] M. Li, H. X. Tang, and M. L. Roukes, Nat. Nanotechnol. 2, 114 (2007).

[14] M. H. Matheny, L. G. Villanueva, R. B. Karabalin, J. E. Sader, and M. L. Roukes, Nano. Lett. 13, 1622 (2013).

[15] H. J. R. Westra, D. M. Karabacak, S. H. Brongersma, M. Crego-Calama, H. S. J. van der Zant, and W. J. Venstra, Phys. Rev. B 84, 134305 (2011).

[16] R. B. Karabalin, L. G. Villanueva, M. H. Matheny, J. E. Sader, and M. L. Roukes, Phys. Rev. Lett. 108, 236101 (2012).

[17] I. Mahboob and H. Yamaguchi, Nat. Nanotechnol. 3, 275 (2008).

[18] D. S. Greywall, B. Yurke, P. A. Busch, A. N. Pargellis, and R. L. Willett, Phys. Rev. Lett. 72, 2992 (1994).

[19] L. G. Villanueva, E. Kenig, R. B. Karabalin, M. H. Matheny, R. Lifshitz, M. C. Cross, and M. L. Roukes, Phys. Rev. Lett. 110, 177208 (2013).

[20] E. Kenig, M. C. Cross, R. Lifshitz, R. B. Karabalin, L. G. Villanueva, M. H. Matheny, and M. L. Roukes, Phys. Rev. Lett. 108, 264102 (2012).

[21] L. G. Villanueva, R. B. Karabalin, M. H. Matheny, E. Kenig, M. C. Cross, and M. L. Roukes, Nano Lett. 11, 5054 (2011).

[22] D. Antonio, D. H. Zanette, and D. López, Nat. Commun. 3, 806 (2012).

[23] M. S. Hanay, S. Kelber, A. K. Naik, D. Chi, S. Hentz, E. C. Bullard, E. Colinet, L. Duraffourg, and M. L. Roukes, Nat. Nanotechnol. 7, 602 (2012).

[24] I. Bargatin, E. B. Myers, J. S. Aldridge, C. Marcoux, P. Brianceau, L. Duraffourg, E. Colinet, S. Hentz, P. Andreucci, and M. L. Roukes, Nano Lett. 12, 1269 (2012).

[25] M. Li, E. B. Myers, H. X. Tang, S. J. Aldridge, H. C. McCaig, J. J. Whiting, R. J. Simonson, N. S. Lewis, and M. L. Roukes, Nano Lett. 10, 3899 (2010).

[26] D. Rugar, R. Budakian, H. J. Mamin, and B. W. Chui, Nature (London) 430, 329 (2004).

[27] S.-B. Shim, M. Imboden, and P. Mohanty, Science 316, 95 (2007).

[28] M.Zhang, G. S. Wiederhecker, S. Manipatruni, A. Barnard, P. McEuen, and M. Lipson, Phys. Rev. Lett. 109, 233906 (2012).

[29] See Supplemental Material at http://link.aps.org/supplemental/ 10.1103/PhysRevLett.112.014101 for the theoretical derivations, experimental and calibration methods, device parameters, and examinations of previous works in NEMS synchronization.

[30] D. G. Aronson, G. B. Ermentrout, and N. Kopell, Physica (Amsterdam) 41D, 403 (1990).

[31] M. C. Cross, J. L. Rogers, R. Lifshitz, and A. Zumdieck, Phys. Rev. E 73, 036205 (2006).

[32] M. C. Cross, A. Zumdieck, R. Lifshitz, and J. L. Rogers, Phys. Rev. Lett. 93, 224101 (2004).

[33] R. Adler, Proceedings of the IRE 34, 351 (1946).

[34] S. H. Strogatz, Nonlinear Dynamics and Chaos: With Applications to Physics, Biology, Chemistry, and Engineering (Studies in Nonlinearity) (Westview Press, Boulder, CO, 2001).

[35] A. Demir, A. Mehrotra, and J. Roychowdhury, IEEE Trans. Circuits Syst. I 47, 655 (2000).

[36] D. K. Agrawal, J. Woodhouse, and A. A. Seshia, Phys. Rev. Lett. 111, 084101 (2013). 